\author{
R. Reifarth ${ }^{\mathrm{a}, *}$, R.C. Haight ${ }^{\mathrm{a}}$, M. Heil ${ }^{\mathrm{b}}$, F. Käppeler ${ }^{\mathrm{b}}$, \\ D.J. Vieira ${ }^{a}$ \\ ${ }^{a}$ Los Alamos National Laboratory, Los Alamos, New Mexico, 87545, USA \\ ${ }^{\mathrm{b}}$ Forschungszentrum Karlsruhe, Institut für Kernphysik, Postfach 3640, D-76021 \\ Karlsruhe, Germany
}

\title{
Neutron capture measurements at a RIA-type facility
}

\begin{abstract}
Neutron capture cross sections of unstable isotopes are important for neutron induced nucleosynthesis as well as for technological applications. The Rare Isotope Accelerator (RIA) or comparable facilities will be able to produce radioactive ion beams up to $10^{12}$ particles/s and would therefore be a suitable place for $(\mathrm{n}, \gamma)$ studies on radioactive isotopes with half-lives between days and months. We propose a facility for measurements of $(\mathrm{n}, \gamma)$ cross sections of unstable isotopes in the $\mathrm{keV}$ range suited for minimal sample masses down to $10^{15}$ atoms, corresponding to minimum half-lives of only $10 \mathrm{~d}$.
\end{abstract}

Key words: keV neutron capture, short-lived samples, radioactive beams, RIA, PACS: 07.05.Fb, 25.40.Lw, 29.25.Rn, 29.40.Vj

\section{Introduction}

In astrophysics the neutron energy range between $1 \mathrm{keV}$ and $1 \mathrm{MeV}$ is most important, because it corresponds to the temperature regimes of the relevant sites for synthesizing all nuclei between iron and the actinides. In this context $(\mathrm{n}, \gamma)$ cross sections for unstable isotopes are requested for the s-process related to stellar helium burning as well as for the $r$ - and p-processes related to explosive nucleosynthesis in supernovae. In the s process, these data are required for analyzing branchings in the reaction path, which can be interpreted as diagnostic tools for the physical state of the stellar plasma. Most

* Corresponding author:

Email address: reifarth@lanl.gov (R. Reifarth).

$U R L$ : www.reifarth.com (R. Reifarth). 
of the nucleosynthesis reactions during the $\mathrm{r}$ and $\mathrm{p}$ processes occur outside the stability valley, involving rather short-lived nuclei. Here, the challenge for $(n, \gamma)$ data is linked to the freeze-out of the final abundance pattern, when the remaining free neutrons are captured as the temperature drops below the binding energy. Since many of these nuclei are too short-lived to be accessed by direct measurements it is, therefore, essential to obtain as much experimental information as possible off the stability line in order to assist theoretical extrapolations of nuclear properties towards the drip lines.

Apart from the astrophysical motivation there is continuing interest on neutron cross sections for technological applications, i.e. with respect to the neutron balance in advanced reactors, which are aiming at high burn-up rates, as well as for concepts dealing with transmutation of radioactive wastes.

The Rare Isotope Accelerator (RIA) (1) will produce radioactive ion beams up to $10^{12}$ particles/s and would, therefore, be the ideal place for neutron capture studies on short-lived radioactive isotopes with half-lives between days and months. Conducting this kind of experiments directly at RIA eases many technical and organizational problems, in particular the depletion of enriched samples by decay in transport, but also safety issues as well as sample handling.

\section{Simulations}

All the discussed processes were aided by detailed Monte-Carlo simulations. The program used was the GEANT 3.21 package (2), developed at CERN in combination with the GCALOR interface (3), which is especially suited for tracking neutrons of thermal energies up to the $\mathrm{MeV}$ range. The simulations including the $\mathrm{BaF}_{2}$ crystal ball are based on the DANCE array, a soccer ball design with 162 crystals to cover the entire solid angle (4; ; 5$)$. As it will become clear in later sections, one crystal needs to be left out to leave space for a proton pipe, which means that all the simulations contained have been carried out with 161 crystals. More details on the simulation technique can be found in Refs. (6; 7; 8).

\section{Experimental Approach}

Measurements on radioactive isotopes represent a stringent challenge for further improvements of experimental techniques. This holds for the neutron sources as well as for the detection systems. Though the activation method or accelerator mass spectroscopy of the reaction products could be applied in a limited number of cases (see e.g. $(9 ; 10)$ ), this report concentrates on the 
more universal method of detecting the prompt capture gamma-rays, which is required for the application of neutron time-of-flight (TOF) techniques.

In this paper we discuss the possibility for using an optimized low energy accelerator as a neutron source for a "Neutron capture program" at RIAtype facilities in combination with a highly segmented, high-efficiency $\gamma$-ray calorimeter.

\subsection{Neutron Source}

Since spallation or photo-neutron sources require large accelerators, it is assumed that a small accelerator is best suited for neutron experiments at RIA. This solution has the additional advantage that the neutron spectrum can be tailored to the specific energy range of interest.

The following discussion is therefore focused on the use of $(p, n)$ reactions for neutron production. Among the different options for producing neutrons in the $\mathrm{keV}$ region, the ${ }^{7} \mathrm{Li}(\mathrm{p}, \mathrm{n}){ }^{7} \mathrm{Be}$ reaction with a threshold of $1.881 \mathrm{MeV}$ is by far the most prolific. Near threshold, one can also take advantage of the fact that kinematically collimated neutrons can be produced in the energy range up to $100 \mathrm{keV}$. A typical neutron spectrum derived on a regular basis during activation measurements at the Research Center Karlsruhe (FZK) is shown in Fig. 1.

\subsection{Proton Accelerator}

Optimized, single ended Van-de-Graaff type accelerators are capable to produce pulsed proton beams with repetition rates between $100 \mathrm{kHz}$ and $5 \mathrm{MHz}$. At present, the best performance of these machines allows one to achieve peak currents of $14 \mathrm{~mA}$ at $1 \mathrm{MHz}$ repetition rate and $0.7 \mathrm{~ns}$ pulse width, corresponding to average beam currents of up to $10 \mu \mathrm{A}$. Nevertheless, for easier comparison all values in the following tables and pictures are simply normalized to $10^{7}$ protons, corresponding to a single pulse at $1.6 \mu \mathrm{A}$ beam intensity and $1 \mathrm{MHz}$ repetition rate. Bombarding a thin metallic Li target just above the neutron production threshold, such a pulse would provide a yield of $1.6 \times 10^{-6}$ neutrons/proton, equivalent to 16 neutrons/pulse (13). A proton current of $10 \mu \mathrm{A}$ with $2 \mathrm{MeV}$ energy implies a heat load at the target of only $20 \mathrm{~J}$, which can be cooled with a jet of compressed air. If a more powerful water cooling should be necessary, it needs to be designed to cool the target outside the flight path of the neutrons towards the sample. This ensures an undisturbed neutron spectrum. 
With with an optimized source, an integral source strength of $10^{8}$ neutrons/s is obtained in the energy range from 3 to $100 \mathrm{keV}$, which is the most relevant temperature regime for neutron capture nucleosynthesis. This neutron spectrum would be produced with a proton energy adjusted $30 \mathrm{keV}$ above threshold and is kinematically collimated into an emission cone of 120 degrees opening angle. At higher proton energies this collimation effect vanishes and neutrons will be emitted into the entire solid angle.

The potential of this neutron source can be illustrated by comparison with the LANSCE facility, the presently most powerful neutron source in the keVregion. For the new neutron capture experiment DANCE the neutron flux between 3 and $100 \mathrm{keV}$ is expected to be $5 \times 10^{5}$ neutrons $/ \mathrm{s} / \mathrm{cm}^{2}$, however at a more favorable duty cycle of $20 \mathrm{~Hz}$.

Possible improvements of the current low energy accelerators appear feasible in several respects, for example by pulsing the extraction from the ion sources, by using pulsed dynamitrons, or by the addition of a proton storage ring, which would result in a further reduction of the repetition rate while keeping the average proton current.

\section{$3.3 \gamma$-Detector}

In neutron measurements on radioactive samples the $\gamma$-ray detection system has to meet a number of requirements:

1) $100 \%$ efficiency for detecting small event rates and for background discrimination via the total $\gamma$-ray energy released per event, 2) fast timing in order to achieve acceptable time-of-flight (TOF) resolution at short flight paths and to minimize pile-up effects, 3) high granularity to reduce the count rate per module and to apply multiplicity cuts for background discrimination, 4) good energy resolution to separate neutron captures on the sample from neutron captures on impurities with different total $\gamma$-ray energy, 5) low neutron sensitivity to avoid excessive backgrounds from scattered neutrons.

These requirements are best met by the $4 \pi \mathrm{BaF}_{2}$ arrays at FZK (14) and LANL (5) with 42 and 162 detector modules, respectively. BaF $F_{2}$ turned out to be the most suited scintillator material because of the unique combination of fast timing, high efficiency, low neutron sensitivity, and good resolution in $\gamma$ -

ray energy. An interesting alternative would be a $4 \pi$ array of $\mathrm{CeF}_{3}$ scintillators (6), but to our knowledge, no large $\mathrm{CeF}_{3}$ crystals are presently available.

As outlined further on, the combination of a high granularity detector similar to the DANCE design and an advanced low energy neutron source appears to represent a promising concept for neutron capture experiments at RIA. 


\subsection{Setup}

The proposed experimental setup is designed for an efficient TOF discrimination of the most important neutron related background components. The main components include

1) a $4 \pi \mathrm{BaF}_{2}$ array for detecting the prompt capture $\gamma$-rays,

2) a high performance proton accelerator with the beam for neutron production ending in the center of the $\mathrm{BaF}_{2}$ array,

3) a very short flight path of only $4 \mathrm{~cm}$ between neutron target and sample.

The schematic drawing of the proposed setup in Fig. 2 indicates the underlying concept and shows the essential dimensions, i.e. an inner diameter of the $\mathrm{BaF}_{2}$ array of $34 \mathrm{~cm}$ and the $4 \mathrm{~cm}$ distance between neutron target and sample.

\subsection{Expected TOF-spectra}

The schematic TOF spectrum in Fig. 3 has been constructed under the following assumptions:

- 1 ns overall time resolution (proton pulse folded with $\mathrm{BaF}_{2}$ resolution),

- $4 \mathrm{~cm}$ neutron flight path between ${ }^{7} \mathrm{Li}$ target and sample,

- $17 \mathrm{~cm}$ inner radius of the $\mathrm{BaF}_{2}$ array

- $100 \mathrm{keV}$ maximum neutron energy.

It has been experimentally shown that a time resolution of $1 \mathrm{~ns}$ can be achieved with the Karlruhe $4 \pi \mathrm{BaF}_{2}$ detector, which has a similar geometry like the one discussed here.

These parameters are sufficient to define the time and energy distributions of the setup sketched in Fig. 2. The neutron energy in keV at the sample position as a function of the neutron time of flight is given by:

$$
E_{\text {neutron }}[\mathrm{keV}]=522.6\left(\frac{s[\mathrm{~cm}]}{t[\mathrm{~ns}]}\right)^{2},
$$

where $\mathrm{s}$ is the flight path in $\mathrm{cm}$ and $\mathrm{t}$ the time of flight in ns.

\subsubsection{Neutron capture on the sample}

The useful TOF window between the prompt $\gamma$-flash and the onset of the background from scattered neutrons can be calculated by the following steps: 
1) The $4 \mathrm{~cm}$ flight path between neutron target and sample is determined by requiring a clear separation of $10 \mathrm{~ns}$ in TOF between the prompt $\gamma$-flash due to the impact of the proton beam and the arrival time of the fastest neutrons $(100 \mathrm{keV})$ at the sample.

2) The background from scattered neutrons starts when the fastest neutrons arrive at the scintillator. These neutrons originate either from scattering at the Li target or at the sample. This occurs at a TOF of $39 \mathrm{~ns}$ when primary neutrons of $5.5 \mathrm{keV}$ energy interact with the sample.

Accordingly, the neutron energy range from $5.5 \mathrm{keV}$ to $100 \mathrm{keV}$ is essentially free of time-dependent background, corresponding exactly to the most relevant part for cross section measurements of astrophysical interest. The $4 \mathrm{~cm}$ flight path implies a reduction in neutron flux compared to the total source strength by about a factor of 10, significantly less compared to the solid angle, because the neutron distribution is strongly forward peaked due to reaction kinematics. This reduction may be compensated by the possible improvements in proton beam intensity as mentioned above. Though the TOF measurement is hampered by the short flight path, the above parameters yield a neutron energy resolution of $12 \%$ at $30 \mathrm{keV}$, assuming that the energy resolution is determined by the TOF resolution. This holds true, if the uncertainty of the flight path is less than $6 \%$.

At energies above $120 \mathrm{keV}$, neutron emission is no longer collimated by reaction kinematics, but becomes increasingly isotropic. In addition, the TOF scale sketched in Fig. 3 is more and more compressed due to the higher neutron velocities, so that separation of the three components becomes more difficult. Measurements in this energy range have to rely on the production of quasi mono-energetic neutrons, where the energy is defined by the thickness of the ${ }^{7} \mathrm{Li}$ target. For neutrons of $500 \mathrm{keV}$ the flight path must be increased to $9 \mathrm{~cm}$ in order to maintain the $10 \mathrm{~ns}$ separation between the prompt $\gamma$-flash and neutron capture events. Assuming, the inner radius of the scintillator would stay at $17 \mathrm{~cm}$, the background from neutron interactions with the scintillator starts already 17 ns past the $\gamma$-flash. Though the useful TOF window is reduced to $7 \mathrm{~ns}$ in this case, it still corresponds to an energy interval between 500 and $200 \mathrm{keV}$. In other words, the method works in principle also at higher energies since the thickness of the Li targets can be defined within a few $\mathrm{keV}$.

Energies below $5.5 \mathrm{keV}$ could be obtained by a combination of shorter flight path, increased inner diameter of the ball, and reduced maximum neutron energy. The latter is possible by reducing the incident proton energy or by bombarding a different neutron production target, like ${ }^{18} \mathrm{O}$. 


\subsubsection{The prompt $\gamma$-flash}

The origin of the so-called prompt $\gamma$-flash are interactions of the proton pulse and of the produced neutrons with the lithium target and the backing. Most of the energy of the protons will be deposited in the backing material. Photons will be produced due to bremsstrahlung, inelastic scattering, and nuclear reactions. In order to reduce the number of photons and the total energy released during a single pulse, a set of simulations have been carried out for different backing materials (Table 1). The corresponding spectra are shown in Figs. 4 to 6 . As a general trend one finds that the total number of emitted $\gamma$-rays as well as the averaged energy per $\gamma$-ray decreases with increasing atomic number of the backing material, if no nuclear interactions are involved (which is true for all investigated backing materials except copper). But even for a backing made of bismuth, the heaviest stable element, the total energy released during one pulse of $10^{7}$ protons is more than $1 \mathrm{GeV}$. Without any further precautions, the detector would be blinded for at least $10 \mathrm{~ns}$.

One way of reducing this $\gamma$-flash is passive shielding. A possible backing and absorber combination made of bismuth is sketched in Figure 7. Bismuth is a good choice for several reasons. First, Table 2 shows that even though the density of bismuth is significantly smaller than that of tungsten, the energy deposited in the surrounding detector is sightly less. Second, bismuth has only one stable isotope with a small average $(\mathrm{n}, \gamma)$ cross section of a few $\mathrm{mb}$ at $\mathrm{keV}$ energies (15), and a small neutron capture Q-value of only $4 \mathrm{MeV}$, in contrast to tungsten and most other elements. Furthermore, there is no inelastic scattering to be expected for the discussed neutron energy range, since the first excited state of ${ }^{209} \mathrm{Bi}$ is at $896.3 \mathrm{keV}$ (16).

This means that most of the neutrons, which will be emitted into such an absorber sphere, will be scattered and eventually leave the sphere without nuclear interaction. Only a very small part will be captured and release at most $4 \mathrm{MeV}$, which is well below the Q-value of most of the isotopes of interest. Depending on the thickness of the absorber, the energy deposition due to the $\gamma$-flash will be reduced by a factor of 2 to 10 and the averaged energy per photon is increased (the spectrum is becoming harder). The resulting spectra for a $\mathrm{Bi}$ absorber with $3 \mathrm{~cm}$ radius are shown in Fig. 8.

If a bismuth absorber would not be possible due to technical reasons, tung-

sten would be the second choice. Therefore, Table 2 contains the results of the simulations also for a tungsten absorber.

\subsubsection{Other neutron-induced reactions}

Most of the neutron-induced reactions can be discriminated via TOF, since the neutrons have to travel at least the distance from the center of the array to the scintillator. But there are three additional sources of background, which could contribute at times, when neutron captures at the sample are registered. 
First, neutrons from the preceding pulse may be captured in the detector with sufficient time delay to cause background events at times, when only captures on the sample are to be expected. Since a repetition rate of $1 \mathrm{MHz}$ corresponds to a pulse spacing of $1 \mu \mathrm{s}$, this so-called wrap-around effect is not negligible as illustrated in Figs. 9 and 10. If the experiment has to be carried out at a repetition rate of $1 \mathrm{MHz}$, further reduction of the background from wraparound neutrons is required. Therefore, the same simulation has been repeated including a spherical ${ }^{6} \mathrm{LiH}$ shell with an inner radius of $10.5 \mathrm{~cm}$ and an outer radius of $16.5 \mathrm{~cm}$. This shell acts as an efficient neutron absorber via ${ }^{6} \mathrm{Li}(\mathrm{n}, \alpha)$, while it is very transparent to $\gamma$-rays (see caption of Fig. 10 for details).

Depending on the neutron TOF, the wrap-around background is reduced by up to two orders of magnitude. If the neutron background level after $1 \mu \mathrm{s}$ of waiting time would still be unacceptably high, the number of wrap-around neutrons could be significantly reduced by choosing a lower repetition rate.

Second, neutrons can get scattered at the different absorber materials and may reach the gold target with a wrong energy-TOF correlation. Fig. 11 shows that this effect is negligible.

Third, neutron captures on the shielding for the prompt $\gamma$-flash, on the neutron absorber, and on the support of the lithium target have to be considered as well. Depending on the materials used, such events can be discriminated via the total energy released. For example, the $\mathrm{Q}$-value of the ${ }^{209} \mathrm{Bi}(\mathrm{n}, \gamma){ }^{210} \mathrm{Bi}$ reaction is $4.606 \mathrm{MeV}$, about $2 \mathrm{MeV}$ below the neutron separation energies of most isotopes near stability. This background reduction based on cuts on the Q-value is discussed in more detail in the following section.

\subsection{Additional Background Discrimination - Expected Energy Spectra}

While almost all beam-related background components can be discriminated via TOF, backgrounds due to neutron interactions with the ${ }^{6} \mathrm{LiH}$ absorber, the $\gamma$-ray shield, the sample backing, inelastic neutron scattering at the sample, sample impurities and from the radioactivity of the sample or due to activations in the detector have to be considered as well. Fig. 12 illustrates the situation for the standard setup of an $0.2 \mathrm{~mm}$ thick gold sample with $0.5 \mathrm{~cm}$ radius, and a $6 \mathrm{~cm}$ thick ${ }^{6} \mathrm{LiH}$ shell around the sample. Events due to neutron capture on gold can clearly be identified via their total deposited energy. These spectra were derived by using only the fast component of the $\mathrm{BaF}_{2}$ scintillation light and a correspondingly lower resolution of the $4 \pi$ array.

In measurements on very small samples it is not always possible to keep neutron captures on the backing or on sample impurities at a negligible level. Since these neutron captures are taking place at the sample position, they exhibit the same time structure as captures on the isotope of interest. However, the almost $100 \%$ detection efficiency for $\gamma$-rays in combination with the high granularity of the proposed detector provides a variety of possibilities for dis- 
criminating these background events. First, the total energy (Q-value) of the reaction depends on the respective isotope. Even though only the fast component of the $\mathrm{BaF}_{2}$ crystals, containing about $20 \%$ of the emitted light, can be used in the proposed setup, the energy resolution at a Q-value of $7 \mathrm{MeV}$, which is characteristic for the $(\mathrm{n}, \gamma)$-reactions of interest, is about $400 \mathrm{keV}$. If the background is dominated by a single isotope, it can be discriminated by the total $\gamma$-ray energy. Second, the average multiplicity of the prompt capture $\gamma$-ray cascade of the isotope under study is usually different from background events. This information is recorded thanks to the high granularity of the $\mathrm{BaF}_{2}$ $\operatorname{array}(7 ; 8)$.

Both options are particularly efficient with respect to the background due to the radioactivity of the sample or activated detector parts as well as inelastic scattering on the sample. In most cases, the total $\gamma$-ray energy released in radioactive decay is less than $2 \mathrm{MeV}$, significantly below average neutron separation energies and is often restricted to multiplicities of less than 3. Inelastic neutron scattering produces a $\gamma$-ray, potentially at the same time as neutron captures would occur. Since the emitted $\gamma$-ray energy needs to be smaller than the neutron energy, it will be below $1 \mathrm{MeV}$ and the multiplicity will be one for most cases. Therefore, both background components can be easily discriminated from neutron captures with typical Q-values of a few $\mathrm{MeV}$.

Any remaining components, e.g. due to ambient background, can be determined by means of background runs without sample or with an empty sample backing.

\section{Comparison with Existing Facilities}

At present, the most sensitive setup for $(\mathrm{n}, \gamma)$ measurements in the $\mathrm{keV}$ region is the DANCE project at LANSCE/LANL (5), which is designed for samples of about $1 \mathrm{mg}$ in mass. Therefore, the proposed setup will only be compared with this facility.

The total number of neutrons per second is directly correlated to the amount of sample material necessary to obtain the required statistics in a given time. Depending on the actual solution, the proposed setup is able to deliver about $10^{7}$ neutrons/s to the sample position, which is 20 times more neutrons than available at DANCE.

Another important aspect concerns the signal-to-background ratio. As mentioned above, the radioactivity of the sample is one background component, that can not be discriminated via time of flight. The number of $\gamma$-rays per time produced via radioactive decay compared to the number of neutron captures per time is, therefore, important. A number of different neutron sources has been investigated by P. Koehler, using essentially this figure-of-merit (17). The following equation shows the ratio of neutron captures per time at the 
possible RIA facility and DANCE, where $\Phi$ stands for the neutron flux, TOF for the lengths of the time-of-flight interval of interest, which is proportional to the flight path, and f for the repetition rate.

$$
\begin{aligned}
\frac{R I A}{D A N C E} & =\frac{\Phi_{R I A}}{\Phi_{D A N C E}} \cdot \frac{T O F_{D A N C E}}{T O F_{R I A}} \cdot \frac{f_{D A N C E}}{f_{R I A}} \\
& =\frac{10^{7}}{5 \cdot 10^{5}} \cdot \frac{20}{0.04} \cdot \frac{20}{10^{6}} \\
& =0.2
\end{aligned}
$$

This implies, that the instantaneous capture rates at both facilities are not too different and can become comparable with the possible improvements of the proton beam intensity.

\section{Count rate and half-life estimates}

Assuming a desired number of $10^{3}$ counts, a neutron flux of $10^{7}$ neutrons $/ \mathrm{s} / \mathrm{cm}^{2}$, an averaged neutron capture cross section of $200 \mathrm{mb}$, and a measuring time of $2 \times 10^{6} \mathrm{~s}$, a sample of $2.5 \times 10^{14}$ atoms would be sufficient for the proposed setup.

Close to the valley of stability, RIA will reach beam intensities of $10^{11}$ to $10^{12}$ particles/s. Accordingly, such samples could be produced within a few hours, presumably even in parallel to normal operation if the collection can run in parasitic mode.

Because of the excellent time resolution of the proposed setup, $\gamma$-rays in the critical energy range above $100 \mathrm{keV}$ can be handled up to a total rate of $20 \mathrm{MHz}$. The limit of $20 \mathrm{MHz}$ for the total rate seen by the array corresponds to a conservatively estimated count rate of $<1 \mathrm{MHz}$ per detector, even if cross talk and cascade transitions are taken into account.

The minimum accessible half-life can be estimated by:

$$
t_{1 / 2}=\frac{\ln 2}{\lambda}=\ln 2 \cdot \frac{N}{A}=\ln 2 \cdot \eta \frac{N}{A_{\gamma}} \approx \eta \cdot 10^{7} \mathrm{~s},
$$

where $t_{1 / 2}$ means the half-life of the isotope under investigation, $\lambda$ the decay constant, $\mathrm{N}$ the number of sample atoms $\left(2.5 \times 10^{14}\right)$, A the sample activity (decays per second), $\eta$ the relative intensity per decay for $\gamma$-rays above 100 $\mathrm{keV}$, and $\mathrm{A}_{\gamma}=\eta \cdot \mathrm{A}=2 \times 10^{7} \mathrm{~s}^{-1}$ the acceptable $\gamma$ activity above $100 \mathrm{keV}$.

Accordingly, for cases with $\eta \leq 0.1$, isotopes with half-lives down to $10 \mathrm{~d}$ can be investigated, consistent with the number of sample atoms available from 
RIA.

\section{Conclusions}

This paper presents a possible way for neutron capture measurements on shortlived isotopes using an existing total absorption calorimeter close to a RIA type facility. While an optimized design will depend on the specific features of the actual facility, this paper attempts to demonstrate that neutron capture measurements in the $\mathrm{keV}$ range with minimal sample masses of $\approx 10^{15}$ atoms are feasible in principle. Depending on the number of $\gamma$-rays emitted per decay, this implies a minimum half-life of the isotope under investigation of only $10 \mathrm{~d}$. Prominent examples, which became accessible with this facility, would be ${ }^{60} \mathrm{Co}$, ${ }^{110} \mathrm{Ag},{ }^{137} \mathrm{Cs},{ }^{147} \mathrm{Nd},{ }^{146} \mathrm{Pm},{ }^{148} \mathrm{Pm},{ }^{170} \mathrm{Tm}$ for analyses of s-process branchings as well as for a variety of isotopes of relevance for explosive nucleosynthesis in the $\mathrm{r}$ and $\mathrm{p}$ processes. Correspondingly, measurements on technologically relevant cross sections could be performed as well, e.g. on ${ }^{237} \mathrm{U}$.

\section{Acknowledgements}

This work has benefited from the use of the Los Alamos Neutron Science Center at the Los Alamos National Laboratory. This facility is funded by the US Department of Energy and operated by the University of California under Contract W-7405-ENG-36.

\section{References}

[1] G. Savard, in: Proc. Particle Accelerator Conference, Chicago, IL, USA, 2001, http://www.phy.anl.gov/ria/publications/ROPA002.pdf.

[2] J. Apostolakis, CERN, Tech. rep., GEANT library, http://wwwinfo.cern.ch/asd/geant/ (1993).

[3] C. Zeitnitz, T.A. Gabriel, Nucl. Instr. Meth. A 349 (1994) 106.

[4] D. Habs, F.S. Stephens, R.M. Diamond, Tech. rep., Report LBL-8945, Lawrence Berkeley Laboratory (1979).

[5] J.B. Wilhelmy, E.P. Chamberlin, M.R. Dragowsky, G.G.G. Miller, P.D. Palmer, L.N. Pangualt, R.S. Rundberg, R.C. Haight, E.H. Seabury, J.L. Ullmann, D.D.D. Strottman, M. Heil, F. Käppeler, R. Reifarth, K. Wisshak, J. Nucl. Science and Technology, Suppl. 2, (2002) 614.

[6] M. Heil, R. Reifarth, F. Käppeler, K. Wisshak, F. Voss, J.L. Ullmann, R.C. Haight, E.H. Seabury, J.B. Wilhelmy, R.S. Rundberg, M.M. Fowler, 
Tech. rep., Report LA-UR-99-4046, Los Alamos National Laboratory (1999).

[7] M. Heil, R. Reifarth, M.M. Fowler, R.C. Haight, F. Käppeler, R.S. Rundberg, E.H. Seabury, J.L. Ullmann, J.B. Wilhelmy, K. Wisshak, F. Voss, Nucl. Instr. Meth. A 459 (2001) 229.

[8] R. Reifarth, M. Heil, F. Käppeler, F. Voss, K. Wisshak, R.C. Haight, M.R. Dragowsky, M.M. Fowler, R.S. Rundberg, J.L. Ullmann, J.B. Wilhelmy, E.H. Seabury, Tech. rep., Report LA-UR-01-4185, Los Alamos National Laboratory (2001).

[9] R. Reifarth, C. Arlandini, M. Heil, F. Käppeler, P.V. Sedychev, A. Mengoni, M. Herman, T. Rauscher, R. Gallino, C. Travaglio, Ap. J. 582 (2003) 1251.

[10] D. Berkovits, E. Boaretto, C. Bordeanu, L.T. Baby, S. Ghelberg, M. Hass, A. Hershkowitz, S.K. Hui, M. Paul, AIP Conf. Proc. 610 (2002) 456.

[11] W. Ratynski, F. Käppeler, Phys. Rev. C 37 (1988) 595.

[12] H. Liskien, A. Paulsen, Atomic Data and Nucl. Data Tables 15 (1975) 57.

[13] R. Reifarth, F. Käppeler, Phys. Rev. C 66 (2002) 054605.

[14] K. Wisshak, K. Guber, F. Käppeler, J. Krisch, H. Müller, G. Rupp, F. Voss, Nucl. Instr. Meth. A 292 (1990) 595.

[15] Z.Y. Bao, H. Beer, F. Käppeler, F. Voss, K. Wisshak, T. Rauscher, Atomic Data Nucl. Data Tables 76 (2000) 70.

[16] R.B. Firestone, Table of Isotopes, Wiley, New York, 1996.

[17] P.E. Koehler, Nucl. Instr. Meth. A 460 (2001) 352. 
Table 1

Number of $\gamma$-rays produced by the interaction of $10^{7}$ protons with different backings (corresponing to one pulse at $\approx 1.6 \mu \mathrm{A}$ and $1 \mathrm{MHz}$ )

Backing Thickness Proton energy Total energy Number of Average $\gamma$-energy

\begin{tabular}{|c|c|c|c|c|c|}
\hline Backing & $(\mathrm{mm})$ & $(\mathrm{MeV})$ & $(\mathrm{GeV})$ & $\gamma$-rays & $(\mathrm{MeV})$ \\
\hline \multirow[t]{3}{*}{$\mathrm{Bi}$} & 0.2 & 1.9 & 1.60 & 3270 & 0.496 \\
\hline & & 2.0 & 1.88 & 3620 & 0.518 \\
\hline & & 2.5 & 3.28 & 5210 & 0.629 \\
\hline \multirow[t]{3}{*}{$\mathrm{Pt}$} & 0.2 & 1.9 & 2.04 & 3540 & 0.577 \\
\hline & & 2.0 & 2.27 & 3790 & 0.598 \\
\hline & & 2.5 & 3.83 & 5410 & 0.708 \\
\hline \multirow[t]{3}{*}{$\mathrm{W}$} & 0.2 & 1.9 & 2.50 & 3680 & 0.679 \\
\hline & & 2.0 & 2.75 & 3930 & 0.698 \\
\hline & & 2.5 & 4.42 & 5500 & 0.803 \\
\hline \multirow[t]{3}{*}{$\mathrm{Ta}$} & 0.2 & 1.9 & 1.75 & 3350 & 0.522 \\
\hline & & 2.0 & 2.05 & 3660 & 0.560 \\
\hline & & 2.5 & 3.70 & 5270 & 0.702 \\
\hline \multirow[t]{3}{*}{$\mathrm{Cu}$} & 0.5 & 1.9 & 12.5 & 4050 & 3.08 \\
\hline & & 2.0 & 13.4 & 4370 & 3.08 \\
\hline & & 2.5 & 20.0 & 6210 & 3.21 \\
\hline \multirow[t]{3}{*}{$\mathrm{Cu}$} & 2.0 & 1.9 & 12.2 & 3950 & 3.08 \\
\hline & & 2.0 & 13.1 & 4230 & 3.09 \\
\hline & & 2.5 & 19.6 & 6080 & 3.21 \\
\hline \multirow[t]{3}{*}{$\mathrm{Li}$} & 0.5 & 1.9 & 3.45 & 7360 & 0.468 \\
\hline & & 2.0 & 3.83 & 8020 & 0.478 \\
\hline & & 2.5 & 6.45 & 12300 & 0.524 \\
\hline \multirow[t]{3}{*}{$\mathrm{Be}$} & 0.5 & 1.9 & 3.12 & 6420 & 0.485 \\
\hline & & 2.0 & 3.46 & 6950 & 0.498 \\
\hline & & 2.5 & 5.66 & 10100 & 0.561 \\
\hline
\end{tabular}


Table 2

Number of $\gamma$-rays produced by the interaction of $10^{7}$ protons with different $\mathrm{W}$ and Bi backings surrounded by a spherical absorber (see Fig. 7).

\begin{tabular}{|c|c|c|c|c|c|c|}
\hline $\begin{array}{l}\text { Backing } \\
\text { Backing }\end{array}$ & $\begin{array}{c}\text { Thickness } \\
\text { (mm) }\end{array}$ & $\begin{array}{l}\text { Sphere Radius } \\
\qquad(\mathrm{mm})\end{array}$ & $\begin{array}{c}\text { Proton energy } \\
\qquad(\mathrm{MeV})\end{array}$ & $\begin{array}{c}\text { Total energy } \\
\qquad(\mathrm{MeV})\end{array}$ & $\begin{array}{c}\text { Number of } \\
\gamma \text {-rays }\end{array}$ & $\begin{array}{c}\text { Average } \gamma \text {-energy } \\
(\mathrm{MeV})\end{array}$ \\
\hline \multirow[t]{3}{*}{ W } & 0.2 & 30 & 1.9 & 155 & 93.5 & 1.66 \\
\hline & & & 2.0 & 191 & 110 & 1.73 \\
\hline & & & 2.5 & 309 & 183 & 1.69 \\
\hline \multirow[t]{3}{*}{$\mathrm{W}$} & 0.2 & 20 & 1.9 & 325 & 255 & 1.27 \\
\hline & & & 2.0 & 354 & 265 & 1.33 \\
\hline & & & 2.5 & 631 & 459 & 1.38 \\
\hline \multirow[t]{3}{*}{ W } & 0.2 & 10 & 1.9 & 805 & 783 & 1.03 \\
\hline & & & 2.0 & 934 & 898 & 1.05 \\
\hline & & & 2.5 & 1610 & 1400 & 1.15 \\
\hline \multirow[t]{3}{*}{$\mathrm{Bi}$} & 0.2 & 30 & 1.9 & 140 & 141 & 0.988 \\
\hline & & & 2.0 & 170 & 165 & 1.03 \\
\hline & & & 2.5 & 394 & 335 & 1.18 \\
\hline \multirow[t]{3}{*}{$\mathrm{Bi}$} & 0.2 & 20 & 1.9 & 274 & 297 & 0.924 \\
\hline & & & 2.0 & 316 & 334 & 0.950 \\
\hline & & & 2.5 & 735 & 683 & 1.08 \\
\hline \multirow[t]{3}{*}{$\mathrm{Bi}$} & 0.2 & 10 & 1.9 & 591 & 753 & 0.785 \\
\hline & & & 2.0 & 699 & 857 & 0.815 \\
\hline & & & 2.5 & 1460 & 1560 & 0.931 \\
\hline
\end{tabular}




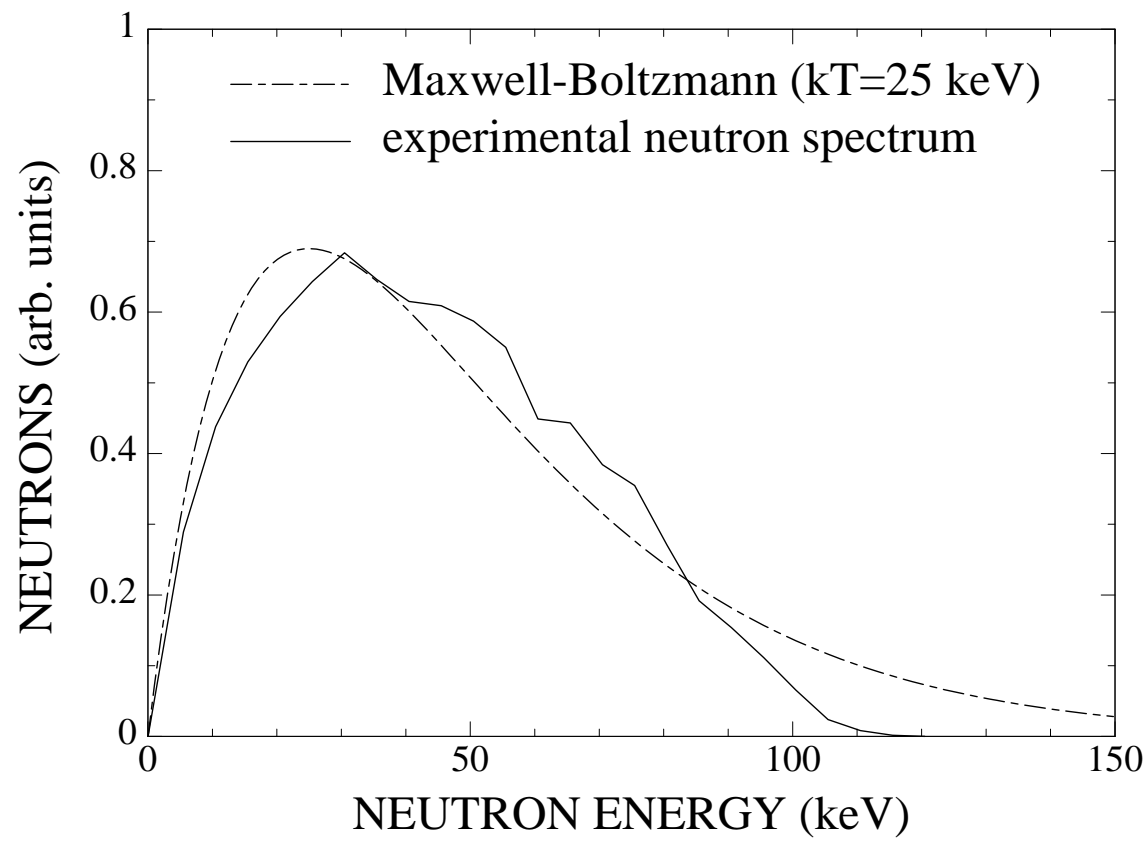

Fig. 1. The neutron spectrum resulting from bombarding of a metallic lithium target with protons of $1912 \mathrm{keV}$ (11). The experimental spectrum (solid line) represents a good approximation of a thermal spectrum for $k T=25 \mathrm{keV}$ (dashed line). More detailed information on the angular dependence of the emitted neutron spectrum can be found in Ref. (12). 


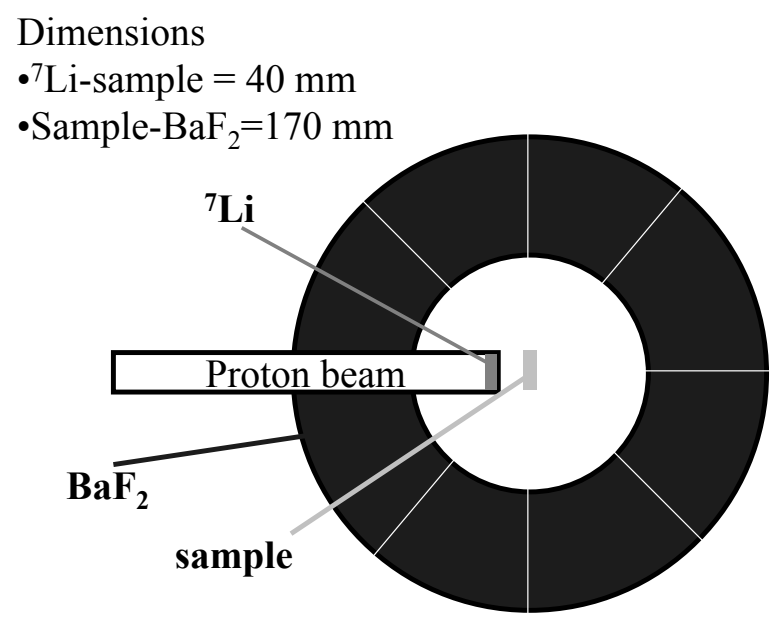

Fig. 2. Schematic sketch of the proposed setup. The segmentation of the crystals is indicated by white lines.

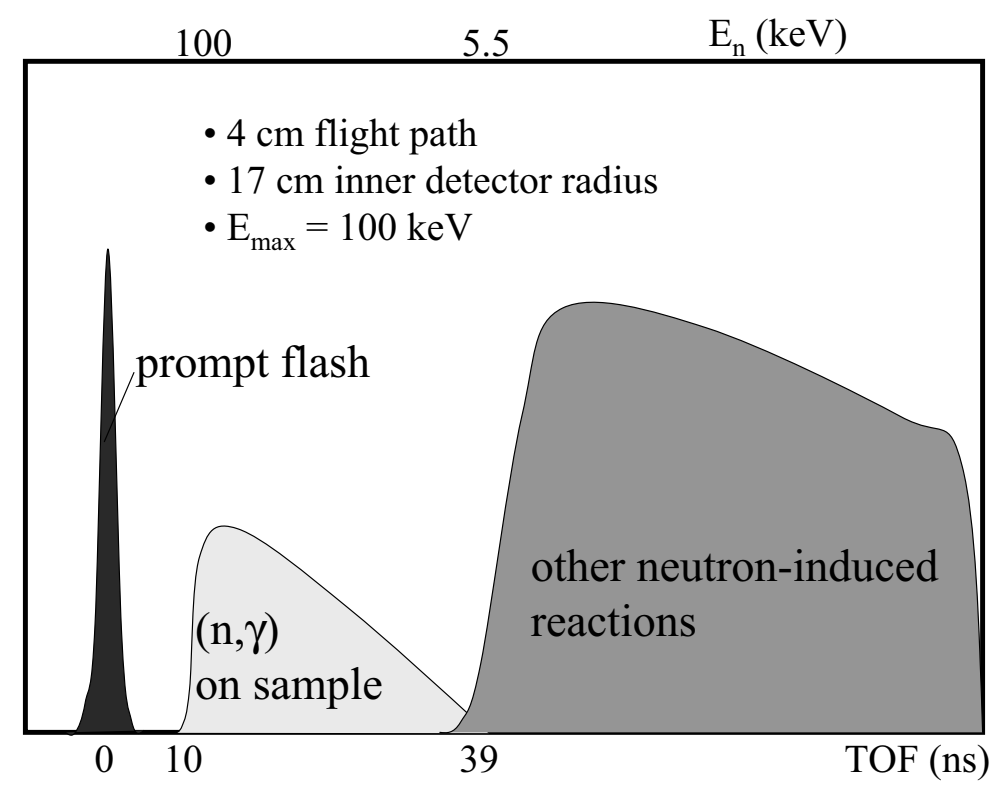

Fig. 3. Schematic TOF spectrum for the setup shown in Fig. 2. 


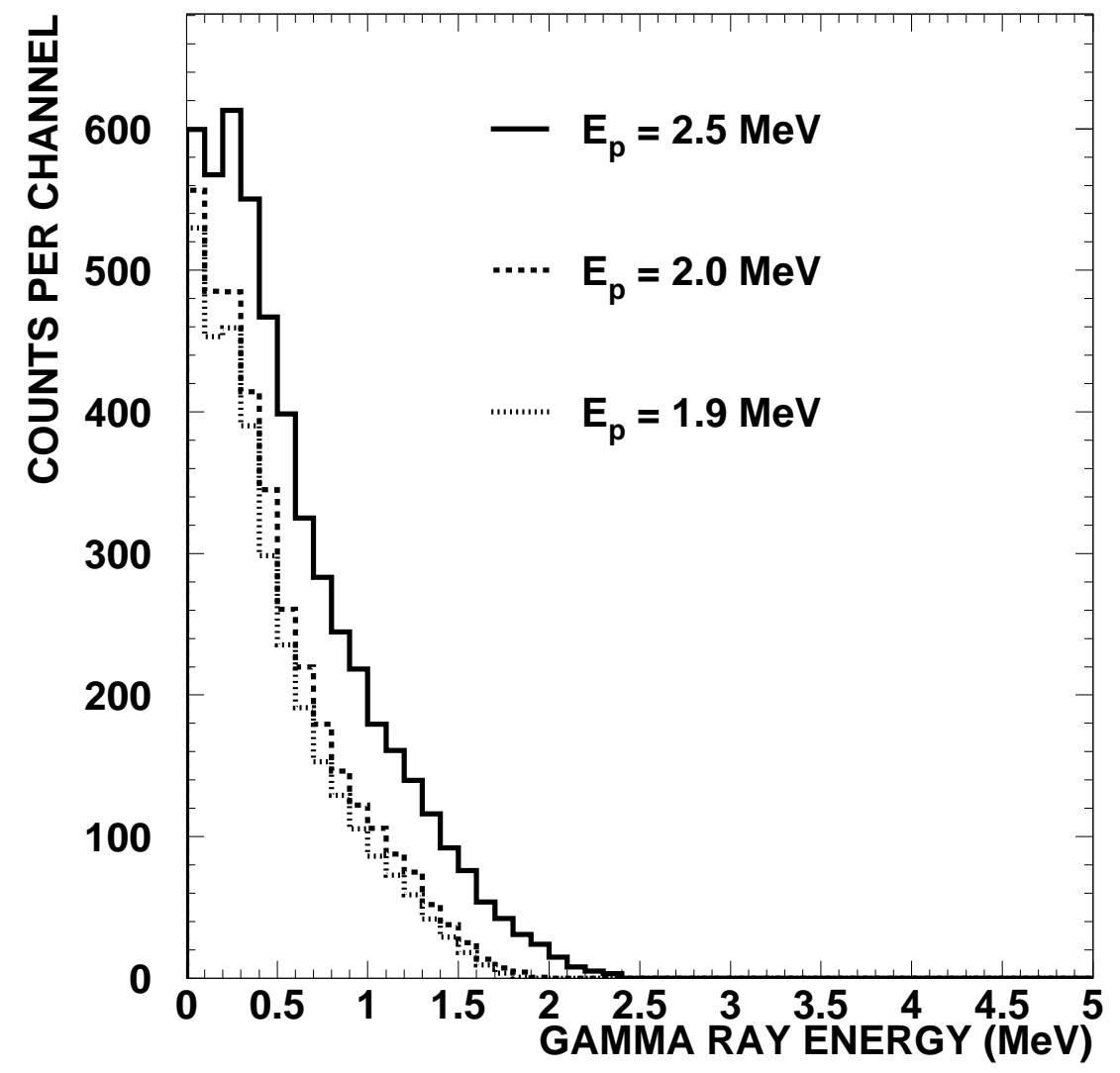

Fig. 4. Interaction of $10^{7}$ protons with a $0.2 \mathrm{~mm}$ Bi target. The histogram binning is $100 \mathrm{keV} /$ channel. 


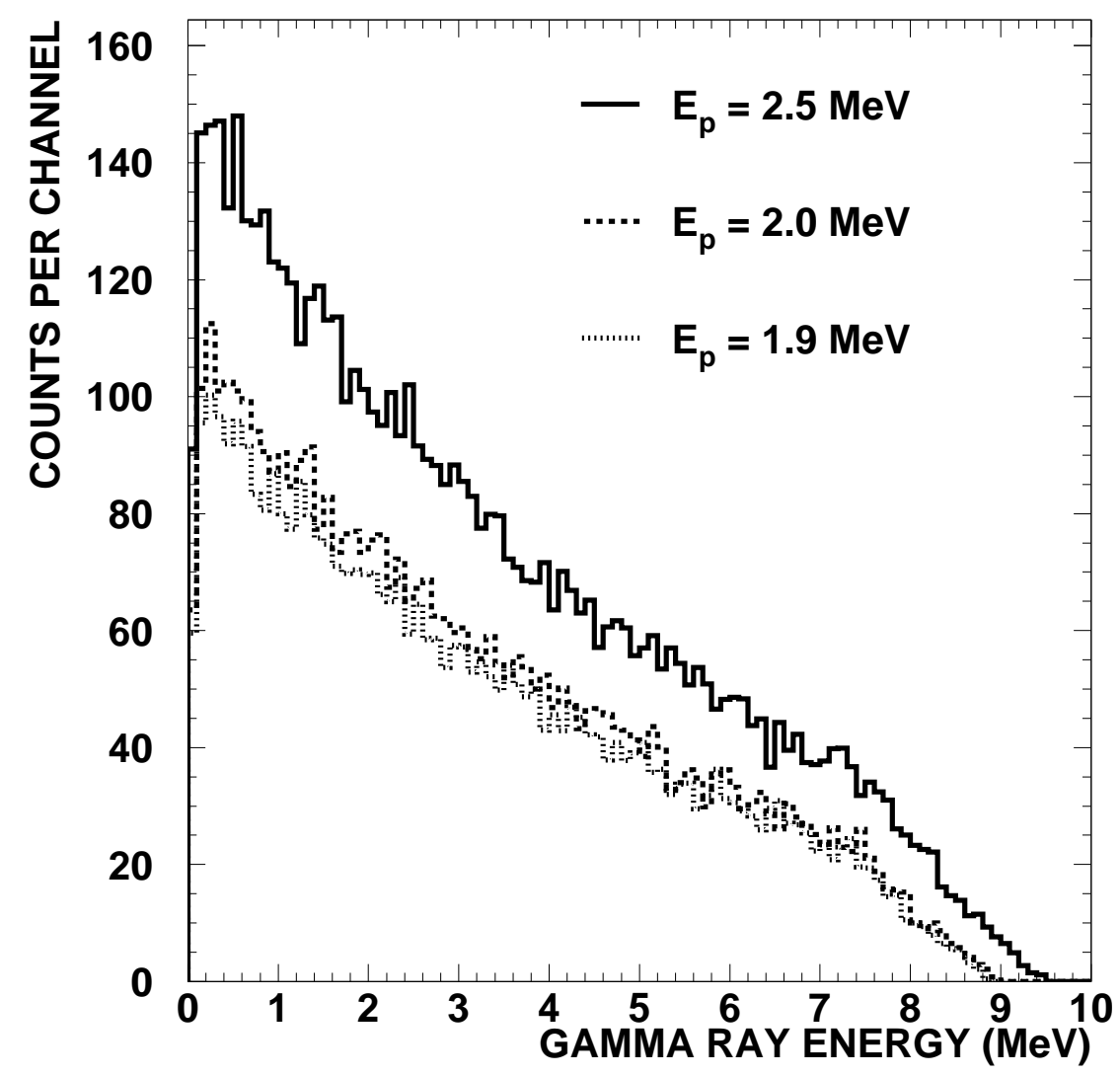

Fig. 5. Interaction of $10^{7}$ protons with a $0.5 \mathrm{~mm} \mathrm{Cu}$ target. Notice the different $\mathrm{x}$-axis compared to Figs. 4 and 6. The histogram binning is $100 \mathrm{keV} /$ channel. 


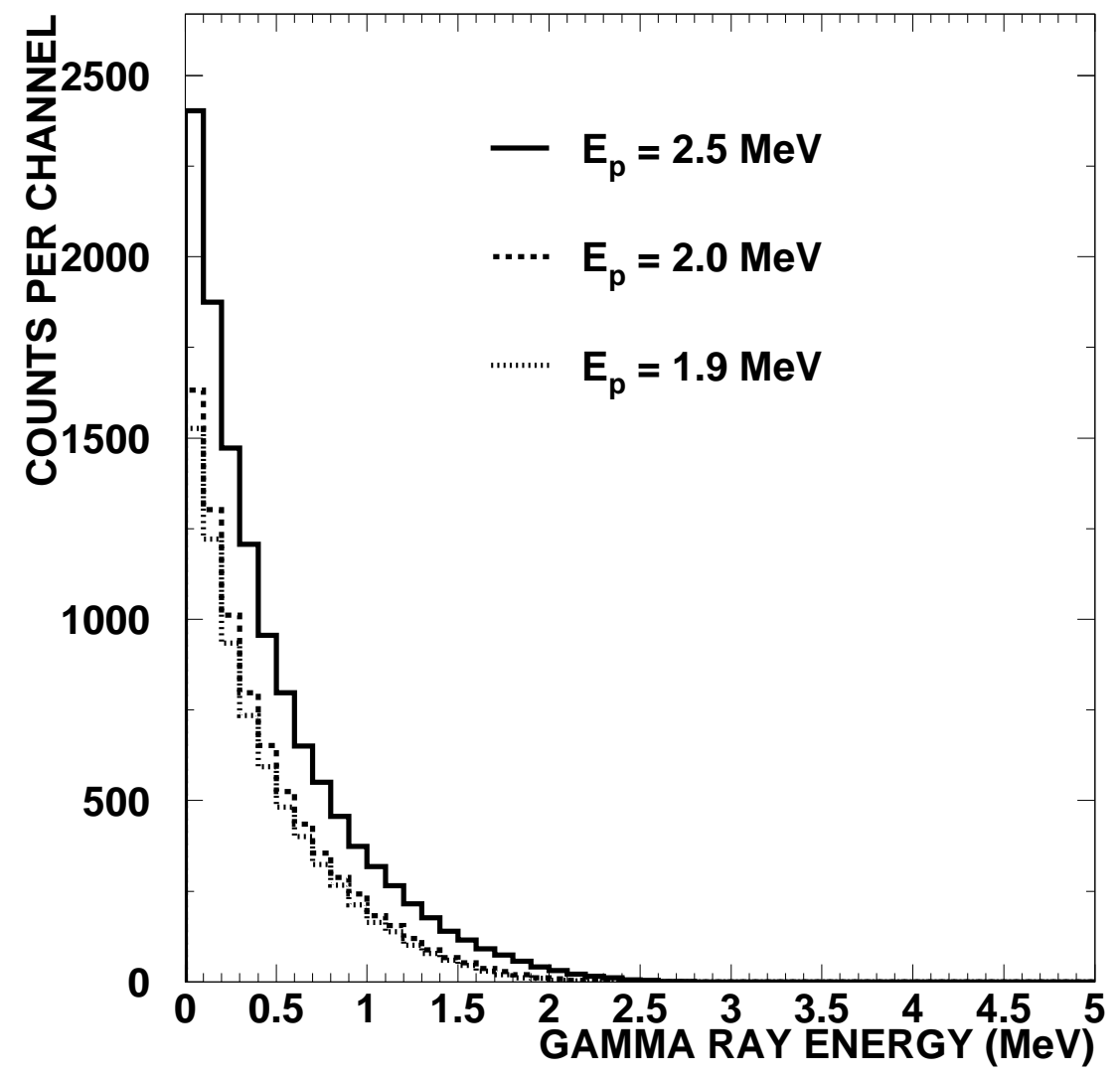

Fig. 6. Interaction of $10^{7}$ protons with a $0.5 \mathrm{~mm}$ Li target. The histogram binning is $100 \mathrm{keV} /$ channel. 


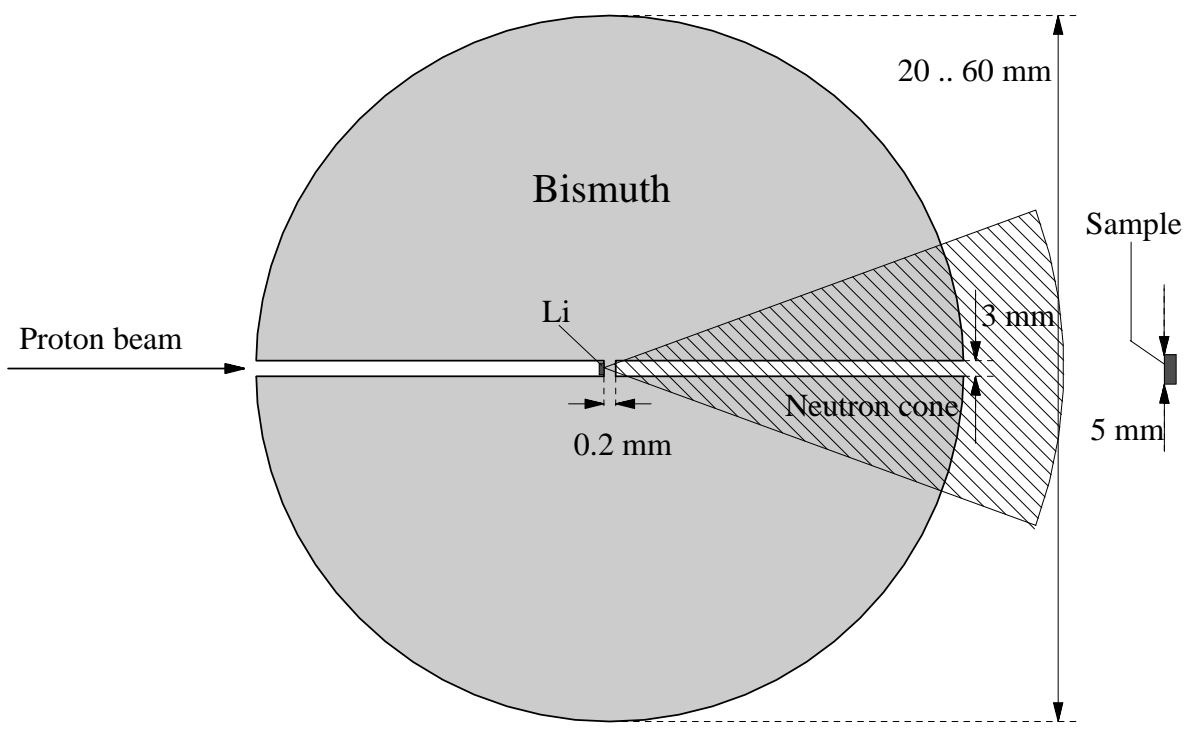

Fig. 7. Schematic view of the simulated spherical Bi targets. The drawing is not on scale. Neutrons are produced via ${ }^{7} \mathrm{Li}(\mathrm{p}, \mathrm{n})$ at a thin lithium layer inside the bismuth absorber sphere. 


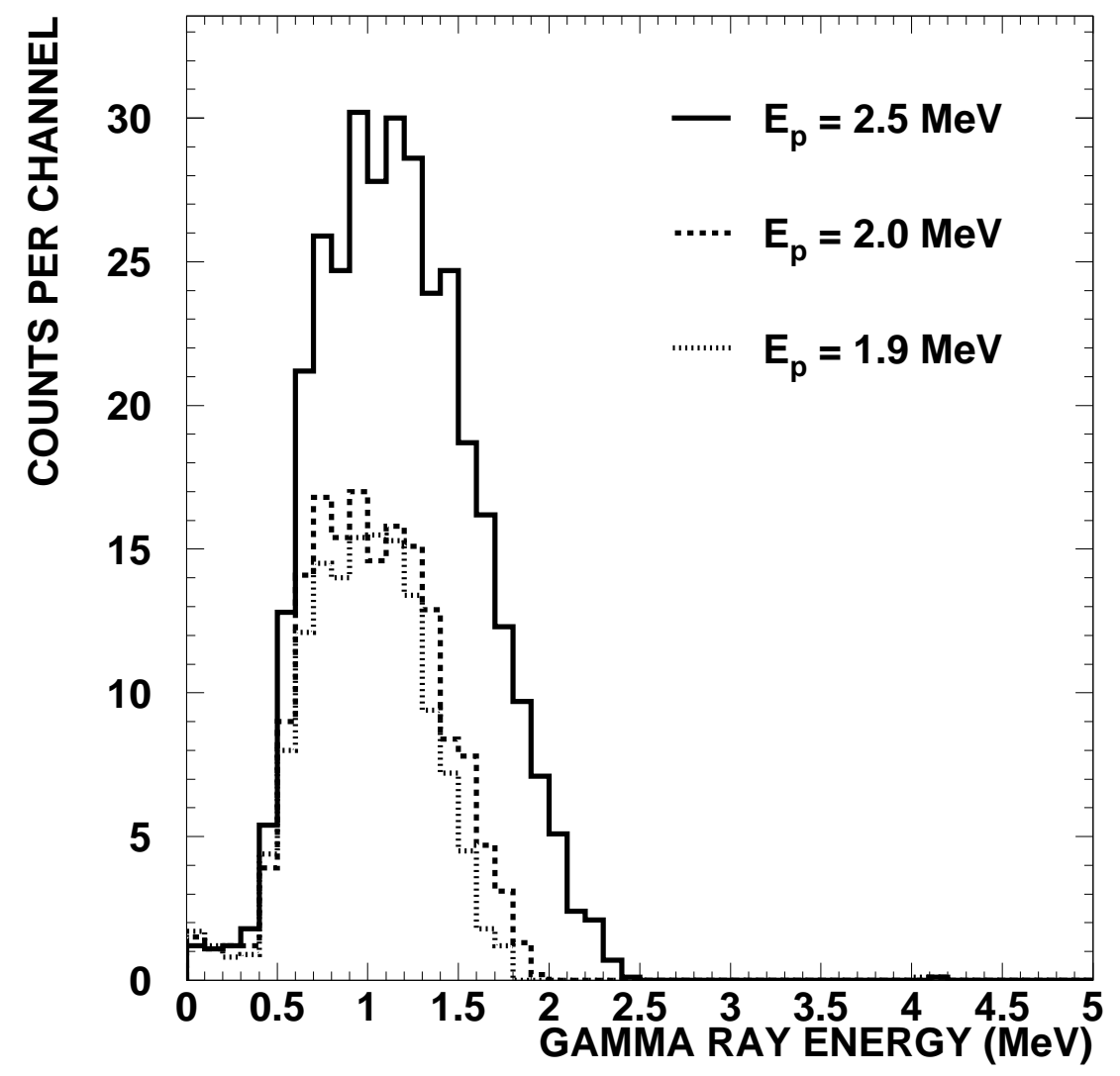

Fig. 8. Interaction of $10^{7}$ protons with an $0.2 \mathrm{~mm}$ Bi target with a surrounding $\mathrm{Bi}$ sphere of $3 \mathrm{~cm}$ radius (see Fig. 7). The histogram binning is $100 \mathrm{keV} /$ channel. 

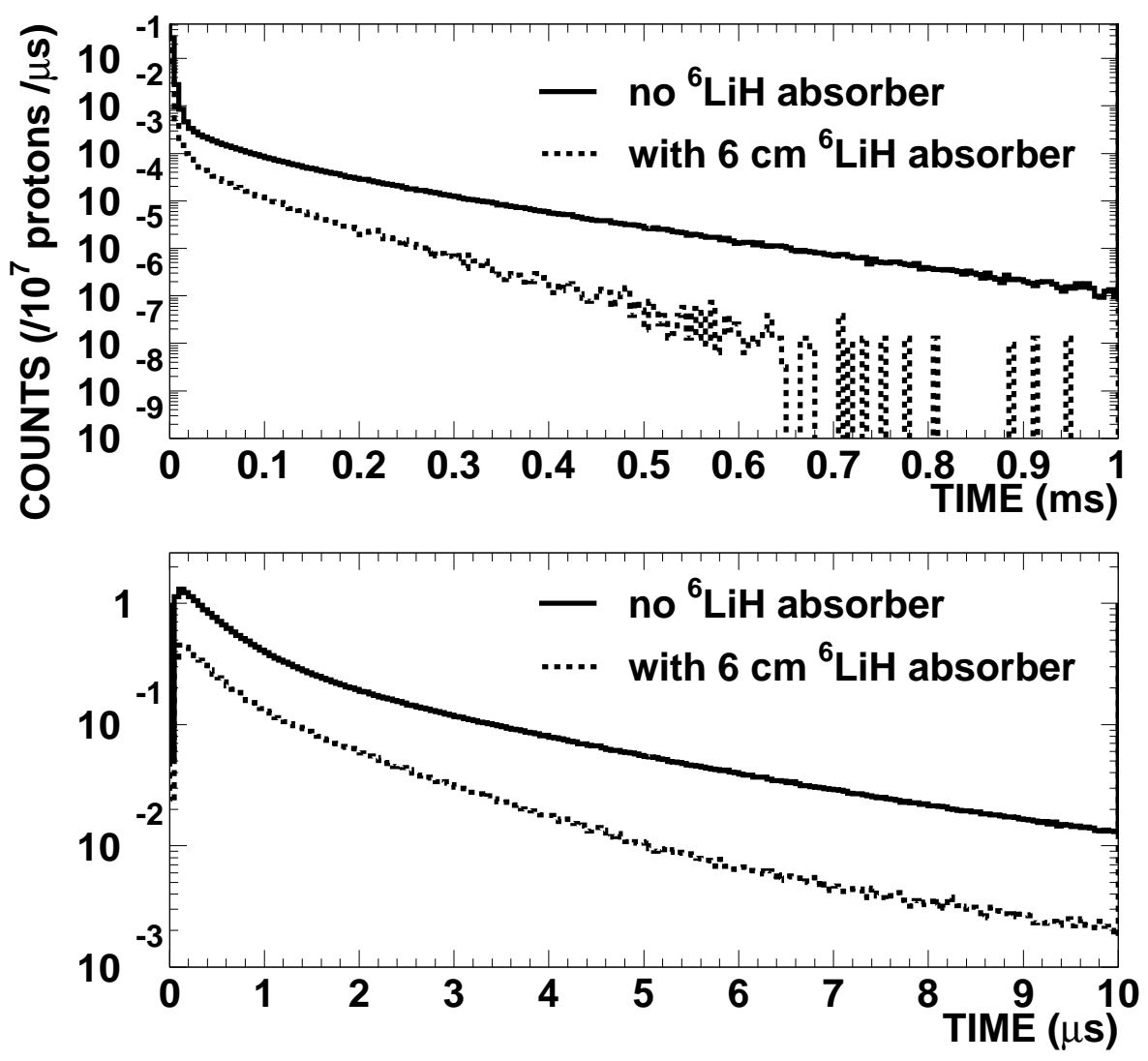

Fig. 9. TOF spectrum for events due to neutron capture in the surrounding material (crystal ball etc.) The spectra have been simulated including a ${ }^{6} \mathrm{LiH}$ neutron absorber shell of $6 \mathrm{~cm}$ thickness around the Li target. The total number of neutrons corresponds to $10^{7}$ protons hitting the metallic Li target. 

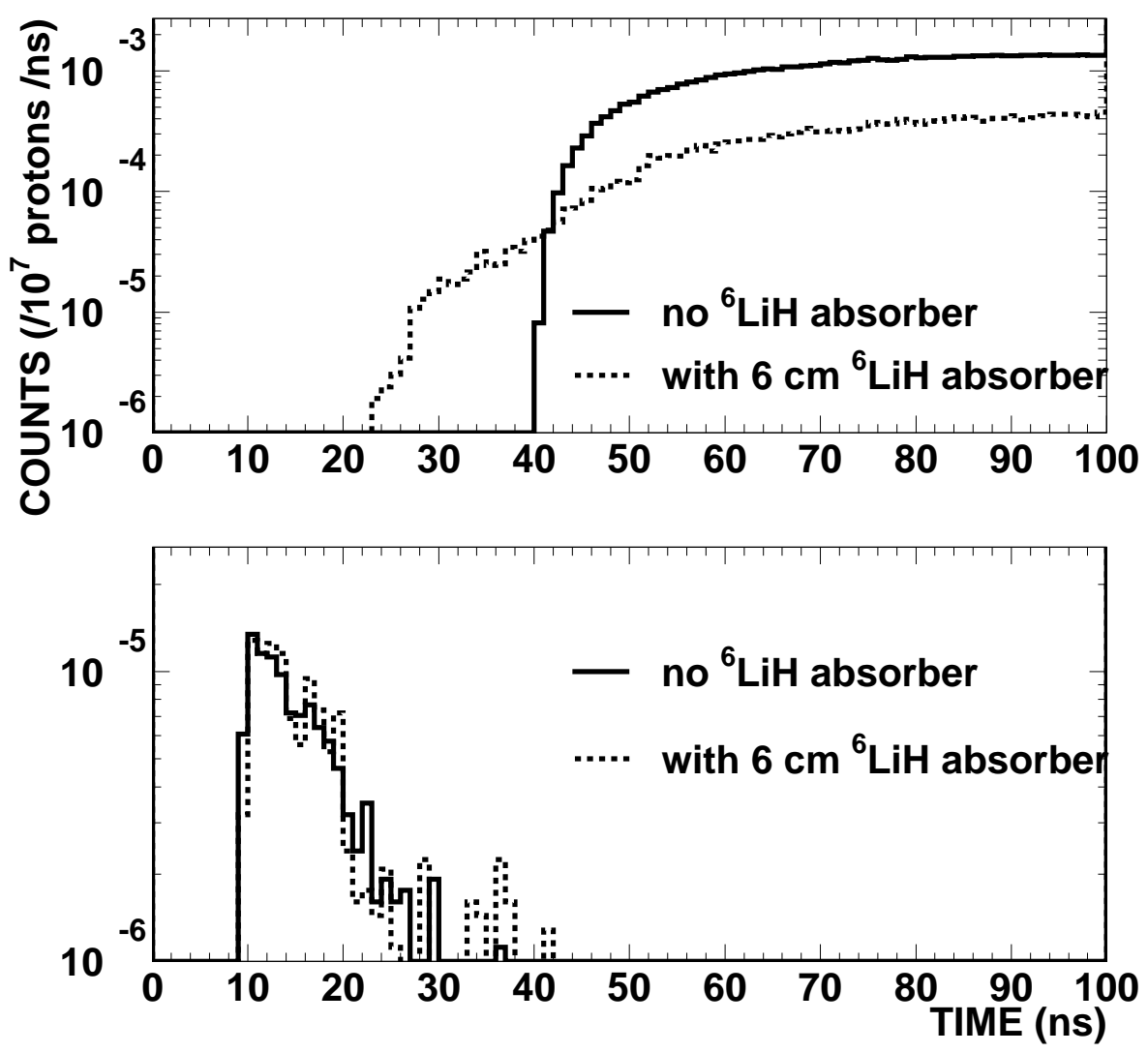

Fig. 10. TOF spectra for events due to neutron capture in the surrounding material (top) and in an $0.2 \mathrm{~mm}$ thick gold sample (bottom). The spectra have been simulated with and without a ${ }^{6} \mathrm{LiH}$ neutron absorber shell of $6 \mathrm{~cm}$ thickness around the $\mathrm{Li}$ target. Accordingly, ${ }^{1} \mathrm{H}(\mathrm{n}, \gamma){ }^{2} \mathrm{H}$ reactions in the ${ }^{6} \mathrm{LiH}$ absorber cause the background of scattered neutrons to start at earlier TOF. This component can be distinguished because its Q-value of $2.2 \mathrm{MeV}$ is considerably lower than the one for gold capture events. As expected, the ${ }^{6} \mathrm{LiH}$ absorber does not affect the gold spectrum. The total number of neutrons corresponds to $10^{7}$ protons hitting the metallic Li target. 


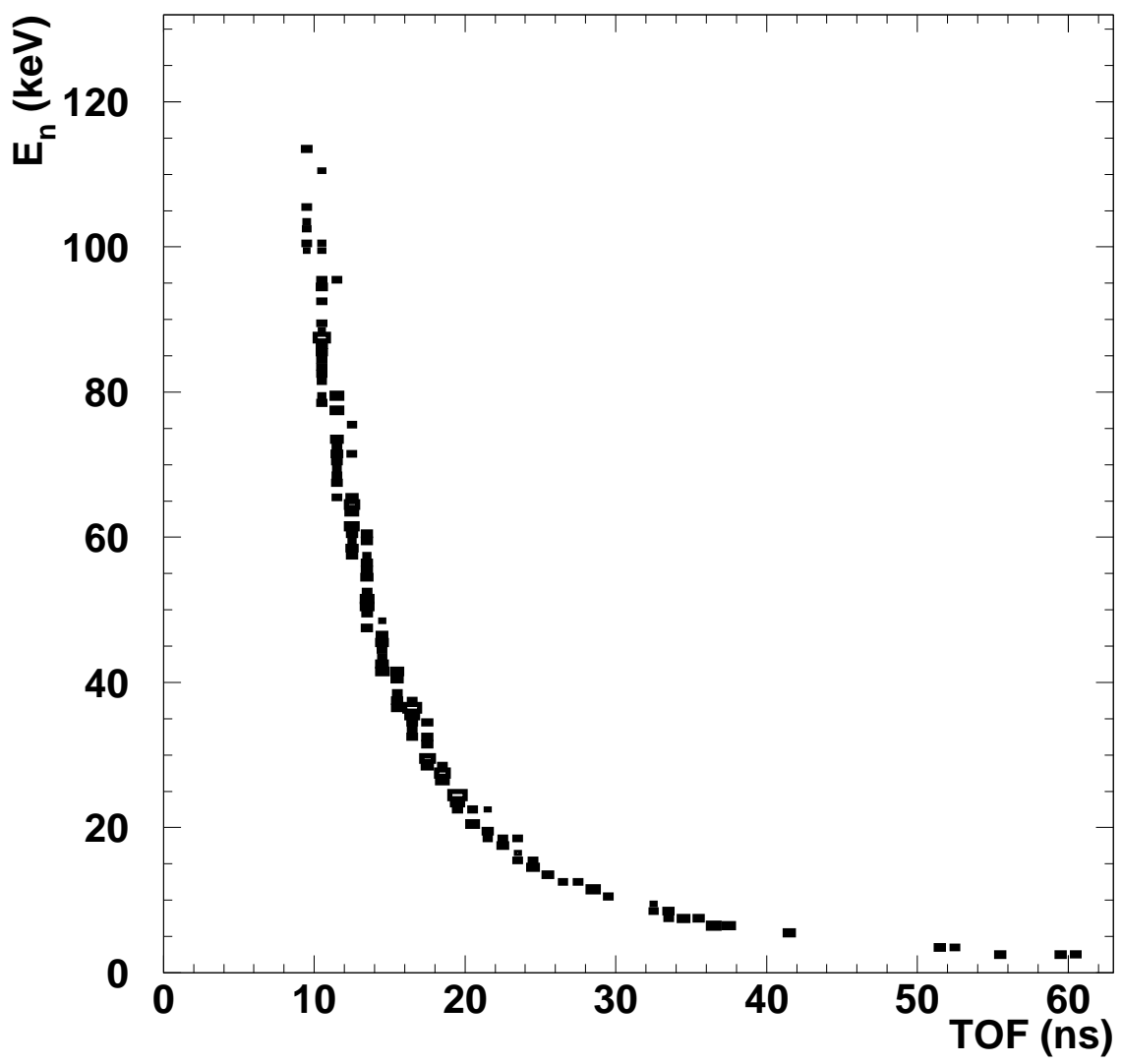

Fig. 11. Neutron energy versus TOF for capture events on the gold sample. All events are following the expected quadratic time-to-energy dependence determined by Eq. (1), thus confirming that the capture contribution of scattered neutrons in the sample is negligible (see text). 


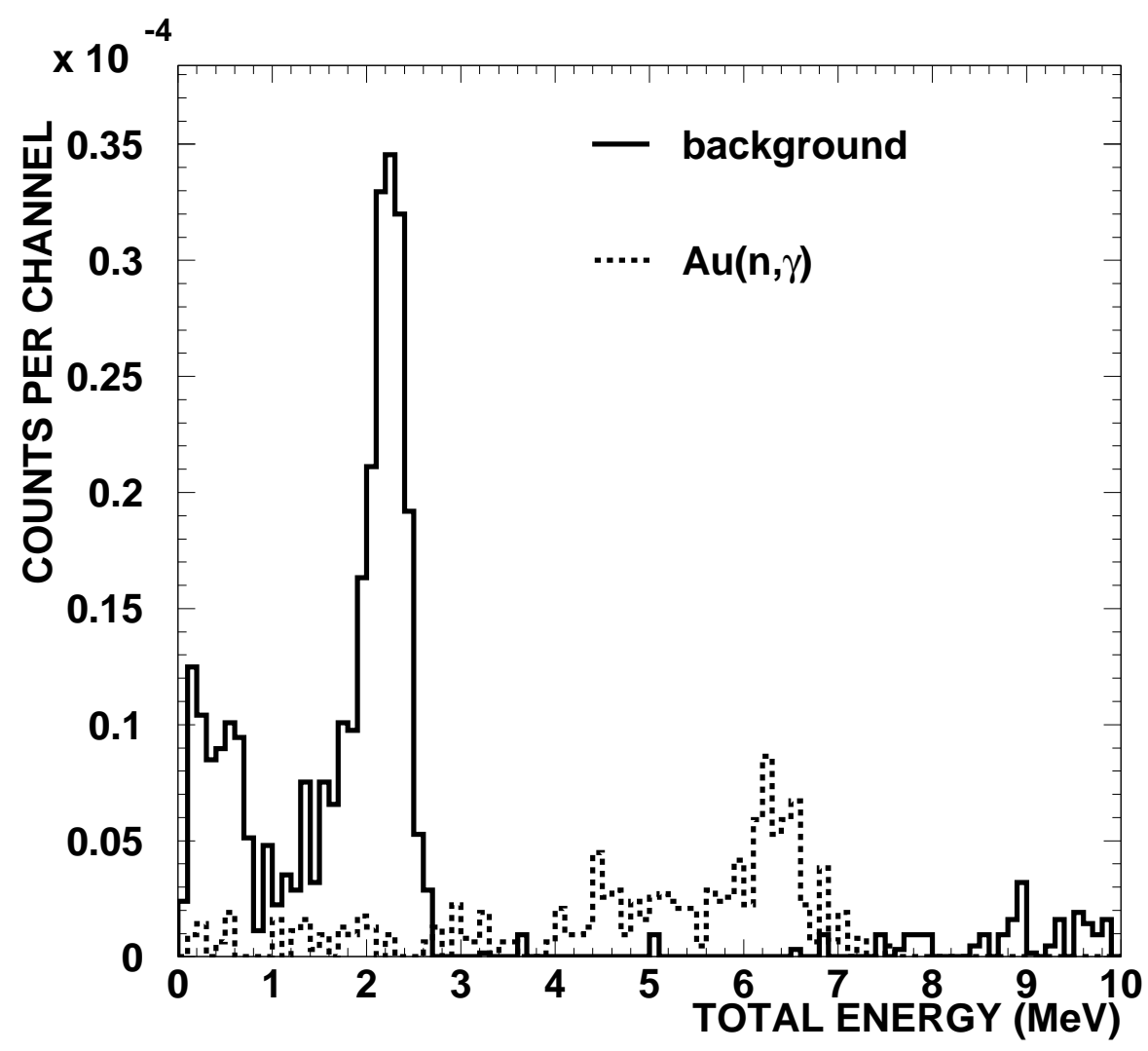

Fig. 12. Total energy deposited in the $4 \pi$ array due to capture in gold (dashed line) and background reactions (solid curve) for the TOF spectrum shown in Fig. (10). The background contains captures on ${ }^{1} \mathrm{H}$ and ${ }^{6} \mathrm{Li}$ as well as inelastic scattering. An energy cut between 3 and $8 \mathrm{MeV}$ will eliminate most of this background. The energy spectrum is shown for a TOF cut of 0-40 ns, which means that captures on the scintillator material are excluded. The total number of neutrons corresponds to $10^{7}$ protons hitting the metallic Li target. The histogram binning is $100 \mathrm{keV} /$ channel. 